\title{
THE INTERNATIONAL MATHEMATICAL OLYMPIAD 1998
}

\author{
Fergus Gaines
}

In 1998, the International Mathematical Olympiad was held in Taipei, Taiwan, from 10th to 21st July. Ireland was represented by a team of six secondary school students-the top six performers in the Irish Mathematical Olympiad, which was held on 9th May 1998. These students were among the initial group of approximately 300 who entered the training programme in November 1997 in centres at UCC, UCD, UL, NUIM and Dundalk IT. The members of the team were:

1. David Conlon, St. Mel's College, Longford.

2. Ciaran Flynn, St. Fintan's High School, Sutton, Co. Dublin

3. Raja Mukherji, Drimnagh Castle CBS, Dublin.

4. Oliver Nash, The High School, Dublin.

5. Darren O’Driscoll, Bandon Grammar School, Cork.

6. Brendan Quigley, Douglas Community School, Cork.

I was the Leader of the team and was accompanied by Kevin Hutchinson of UCD as an Official Observer. Gordon Lessells of the University of Limerick was the deputy leader. Gordon organized the final training camp for the students which took place at the University of Limerick from 29th June to 2nd July. Eleven other students, who had performed well in the Irish Mathematical Olympiad and who will still be eligible to compete in 1999, also took part in the training camp.

\section{The Competition}

Kevin and I arrived in Taiwan on Friday, 10th July and one of the first things we heard was that China, one of the top performing countries in the IMO, would not be sending a team because 
the hosts insisted on using the titles "Taiwan" and "Republic of China" instead of the internationally accepted "China-Taipei". The team leaders and the observers from all of the participating countries were accommodated in a resort area to the north of Taipei, well away from the students' accommodation in National Taiwan Normal University (NTNU). The leaders formed the IMO jury and their initial task was to select the six competition problems from a list of 28 , shortlisted from those submitted by the participating countries. None of the problems submitted by Ireland made it to the short list. The problems on the short list which are not chosen for the IMO remain confidential and may not be published until one year has elapsed. Since the jury consists of the leaders of all the participating countries, the discussions at the meetings can get very heated and acrimonious and national rivalries can rear their ugly heads.

After two days of intense discussion, the six problems and the precise English wording were agreed. The second day's deliberations had taken 12 hours to complete but, about ten minutes after the jury meeting had ended and when a number of people had already left, Professor Svetoslav Savchev, who was a member of the organizers' Problem Selection Committee which drew up the shortlist, announced that he had just discovered that one of the problems selected was very close in content to a problem that had already appeared in the Canadian journal Crux Mathematicorum. After some discussion, it was agreed the problem would have to be withdrawn, because IMO problems are supposed to be completely unseen and several leaders pointed out that many of their students subscribe to Crux. An informal discussion then took place on how best to proceed, but it soon degenerated into chaos since everyone was very tired. However, the next morning, reason prevailed, a substitute was found for the deleted problem, and the papers and their translations into all the languages required by the students were approved by the jury.

The Irish team, accompanied by Gordon Lessells, arrived in Taipei on 12th July but, since they were one day early, did not move into their accommodation in NTNU until the next day. Because the weather was very hot and humid, the students stayed 
indoors most of the time. This lessened the time available to socialize with students from other countries-one of the important features of the IMO. The Official Opening, which was performed by Mr Vincent Siew, the Prime Minister, took place on 14th July.

The students sat the first paper on Wednesday, 15th July, and the second one the following day. Each paper lasted $4 \frac{1}{2}$ hours. After each exam, Gordon was on hand to talk to our students about the questions and to give support to those who felt they hadn't done as well as they expected. On the morning of each exam, Kevin and I travelled with the other leaders to NTNU-a two-hour journey through Taipei's chaotic traffic jams-to be present in the jury room to answer queries from contestants about the papers.

At lunchtime on the day of the second paper, all leaders, deputy leaders and observers transferred to the Howard Plaza Hotel in central Taipei. The Irish students' answer books were carefully read by Gordon, Kevin and me and provisional marks assigned in accordance with the marking scheme supplied by the Taiwanese coordinators. There was quite a delay in getting the students' work because photocopies were made of all the answers by the local IMO organization. This was done for two reasons: to prevent any leader from making additions to the students' work and to give the coordinators the chance to read each student's answers before the coordination sessions. On the Friday and Saturday, we went to the coordinators for each question to finalize the marks to be awarded to each student. A lot of hard work had to be done in advance of this because it was important to have very clear arguments for the marks we thought our students should get. In one case, we were more than pleasantly surprised by the generosity of the coordinators. In another case we had to fight for the marks-the coordinators were quite taken aback when we indicated that one of Raja Mukherji's answers should get full marks. They hadn't understood his argument, but readily gave the marks when we explained it to them. Coordination went quite quickly, mainly due to the fact that the coordinators were well-acquainted with each student's work before we went to see them. 


\section{The Results}

The high point of the IMO for the Irish team in Taiwan was the award of a bronze medal to 15-year old Raja Mukherji. Since Ireland began participating in the IMO in 1988, this is only the third time an Irish student has won a medal-bronze medals were won by Andrew McMurry in 1990 and Kevin McGerty in 1993. Given our limited training programme, we do not expect our students to win medals very often. Raja has been in the training programme for the last three years and has learned a lot of mathematics in that time. He already represented Ireland in the IMO in Argentina in 1997 and will still be eligible to take part in the 40th IMO in Romania in 1999. Each question in the IMO is marked out of 7 marks, making a total of 42 for the whole exam. Medals are awarded to "not more than half the students taking part, at most $1 / 12$ th of the students can win gold, at most $1 / 6$ th silver and at most $1 / 4$ bronze". The cut-off mark for a bronze medal in Taiwan was 14 points, so Raja's total of 18 points gave him a comfortable margin. This was the highest score ever achieved by an Irish student in an IMO and, to put it into perspective, Raja's score equalled the sum of the scores of the other five members of the Irish team! Since each mark awarded represents a substantial contribution towards the solution of a problem, marks are very hard to earn at the IMO. In fact, of the 404 students who took part, 16 scored zero!

The IMO is a competition for individuals, but there is always an unofficial ranking of national teams. The top ten teams this year, in order, were Iran, Bulgaria, Hungary, USA, Republic of China (Taiwan), Russia, Indonesia, Ukraine, Vietnam and Yugoslavia. This order is probably a reflection of the elaborate training programmes organized by some countries. Many countries have a series of mathematical competitions, throughout their educational system and, in some cases, their training programmes rival those for training professional athletes. With our limited programme, we have achieved modest success. This year, the Irish team ranked 56 th of the 74 competing countries.

When not taking part in the competition, the students were taken on several cultural and sight-seeing trips. One trip took 
them to a region in the centre of Taiwan, on the very day that particular area experienced its worst earthquake for many years! Although five people were killed by a collapsing building our students, who were travelling in their coach at the time, never even noticed the 'quake!

The problems in national Olympiads are always somewhat easier than those of the IMO. So, for comparison, we give first the problems of the 1998 Irish Mathematical Olympiad. The time allowed for each paper in the Irish competition is three hours, whereas that in the IMO itself is four and a half hours. Here are the two Irish papers:

\section{Eleventh Irish Mathematical Olympiad}

\section{First Paper}

1. Show that if $x$ is a nonzero real number, then

$$
x^{8}-x^{5}-\frac{1}{x}+\frac{1}{x^{4}} \geq 0 .
$$

2. $P$ is a point inside an equilateral triangle such that the distances from $P$ to the three vertices are 3,4 and 5, respectively. Find the area of the triangle.

3. Show that no integer of the form $x y x y$ in base 10 (where $x$ and $y$ are digits) can be the cube of an integer.

Find the smallest base $b>1$ for which there is a perfect cube of the form $x y x y$ in base $b$.

4. Show that a disc of radius 2 can be covered by seven (possibly overlapping) discs of radius 1 .

5. If $x$ is a real number such that $x^{2}-x$ is an integer, and, for some integer $n \geq 3, x^{n}-x$ is also an integer, prove that $x$ is an integer. 
Second Paper

6. Find all positive integers $n$ that have exactly 16 positive integer divisors $d_{1}, d_{2}, \ldots, d_{16}$ such that

$$
1=d_{1}<d_{2}<\cdots<d_{16}=n
$$

$d_{6}=18$ and $d_{9}-d_{8}=17$.

7. Prove that if $a, b, c$ are positive real numbers, then

$$
\frac{9}{a+b+c} \leq 2\left(\frac{1}{a+b}+\frac{1}{b+c}+\frac{1}{c+a}\right),
$$

and

(2) $\frac{1}{a+b}+\frac{1}{b+c}+\frac{1}{c+a} \leq \frac{1}{2}\left(\frac{1}{a}+\frac{1}{b}+\frac{1}{c}\right)$.

8. Let $\mathbb{N}$ be the set of natural numbers (i.e., the positive integers).

(a) Prove that $\mathbb{N}$ can be written as a union of three mutually disjoint sets such that, if $m, n \in \mathbb{N}$ and $|m-n|=2$ or 5 , then $m$ and $n$ are in different sets.

(b) Prove that $\mathbb{N}$ can be written as a union of four mutually disjoint sets such that, if $m, n \in \mathbb{N}$ and $|m-n|=2,3$ or 5 , then $m$ and $n$ are in different sets. Show, however, that it is impossible to write $\mathbb{N}$ as a union of three mutually disjoint sets with this property.

9. A sequence of real numbers $x_{n}$ is defined recursively as follows: $x_{0}$ and $x_{1}$ are arbitrary positive real numbers, and

$$
x_{n+2}=\frac{1+x_{n+1}}{x_{n}}, n=0,1,2, \ldots
$$

Find $x_{1998}$.

10. A triangle $A B C$ has positive integer sides, $\widehat{A}=2 \widehat{B}$ and $\widehat{C}>90^{\circ}$. Find the minimum length of its perimeter.

The questions of the 39th IMO were as follows. We give solutions to these IMO problems on pp. $72-76$. 
Thirty Ninth International Mathematical Olympiad

$$
\text { First Day — 15th July } 1998
$$

1. In the convex quadrilateral $A B C D$, the diagonals $A C$ and $B D$ are perpendicular and the opposite sides $A B$ and $D C$ are not parallel. Suppose that the point $P$, where the perpendicular bisectors of $A B$ and $D C$ meet, is inside $A B C D$. Prove that $A B C D$ is a cyclic quadrilateral if and only if the triangles $A B P$ and $C D P$ have equal areas.

2. In a competition, there are $a$ contestants and $b$ judges, where $b \geq 3$ is an odd integer. Each judge rates each contestant as either "pass" or "fail". Suppose that $k$ is a number such that, for any two judges, their ratings coincide for at most $k$ contestants. Prove that

$$
\frac{k}{a} \geq \frac{b-1}{2 b}
$$

3. For any positive integer $n$, let $d(n)$ denote the number of positive divisors of $n$ (including 1 and $n$ itself).

Determine all positive integers $k$ such that

$$
\frac{d\left(n^{2}\right)}{d(n)}=k
$$

for some $n$.

$$
\text { Second Day — 16th July } 1998
$$

4. Determine all pairs $(a, b)$ of positive integers such that $a b^{2}+$ $b+7$ divides $a^{2} b+a+b$

5. Let $I$ be the incentre of the triangle $A B C$. Let the incircle of $A B C$ touch the sides $B C, C A$ and $A B$ at $K, L$ and $M$, respectively. The line through $B$ parallel to $M K$ meets the lines $L M$ and $L K$ at $R$ and $S$, respectively. Prove that $\angle R I S$ is acute. 
6. Consider all functions $f$ from the set $\mathbb{N}$ of all positive integers into itself satisfying

$$
f\left(t^{2} f(s)\right)=s(f(t))^{2},
$$

for all $s$ and $t$ in $\mathbb{N}$. Determine the least possible value of $f(1998)$.

Fergus Gaines

Department of Mathematics

University College Dublin

Belfield

Dublin 4 